\title{
BMJ Open Efficacy evaluation of an anti-caries varnish: protocol for a phase II randomised controlled trial
}

\author{
Peter Milgrom, ${ }^{1,2}$ Ohnmar Tut, ${ }^{1}$ Marilynn Rothen, ${ }^{1,3}$ Lloyd Mancl, ${ }^{1}$ Marcelle Gallen, ${ }^{4}$ \\ Jason M Tanzer ${ }^{5}$
}

To cite: Milgrom $P$, Tut 0 , Rothen $\mathrm{M}$, et al. Efficacy evaluation of an anticaries varnish: protocol for a phase II randomised controlled trial. BMJ Open 2017;7:e017866. doi:10.1136/ bmjopen-2017-017866

- Prepublication history and additional material are available. To view these files please visit the journal online (http://dx. doi.org/10.1136/bmj-2017017866).

Received 25 May 2017 Accepted 1 June 2017

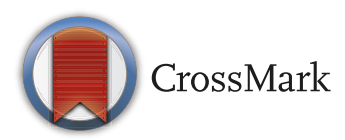

${ }^{1}$ Department of Oral Health Sciences, School of Dentistry, University of Washington, Seattle, Washington, USA ${ }^{2}$ Advantage Silver Dental Arrest, LLC, Redmond, Oregon, USA ${ }^{3}$ Regional Clinical Dental Research Center, Institute of Translational Health Sciences, University of Washington, Seattle, Washington, USA ${ }^{4}$ Department of Dental Services, Pohnpei State Health Department, Kolonia, Federated States of Micronesia

${ }^{5}$ Department of Oral and Maxillofacial Diagnostic Sciences, University of Connecticut Health Center Farmington, Connecticut, USA

Correspondence to

Dr. Peter Milgrom; dfrc@uw.edu

\section{ABSTRACT}

Introduction Dental caries (tooth decay) is a common disease in which the products of sugar metabolism by certain bacteria that populate the tooth surface induce the development and progression of lesions (cavities). This is a phase II single-centre randomised, double-blind, active-controlled, parallel-group trial to assess the efficacy of a combination povidone iodine and sodium fluoride dental varnish to determine if it is superior to a varnish containing only sodium fluoride in the prevention of new caries lesions. The objective of this report is to describe the rationale and protocol for the trial.

Methods and analysis The study site is Pohnpei State, Federated States of Micronesia. The study population is 284 children $48-84$ months old. The primary outcome will be the surface-level primary molar caries increment $\left(\mathrm{d}_{2-3} \mathrm{mfs} / \mathrm{DMFS}\right)$ at 2 years post baseline. The incremental dental caries at 1 year will also be compared between the two interventions. The secondary outcome is the Facial Image Scale after the initial treatment and after the fifth treatment at 1 year that gauges the child's response to the treatment.

Ethics and dissemination The Western Institutional Review Board (designated IRB) and the Institutional Review Board of the College of Micronesia-FSM approved all study procedures. The US Food and Drug Administration (FDA) has issued IND 128835 covering this study. The study results will be published and submitted to the FDA in support of a new drug application.

Trialregistration number NCT03082196.

\section{INTRODUCTION}

Topical fluorides have long been the mainstay in the prevention of dental caries. There are abundant data on sodium fluoride's (NaF) ability to foster remineralisation of tooth enamel. ${ }^{1} \mathrm{NaF}-$ containing tooth varnish preparations are the recommended vehicle for delivering topical fluoride for dental caries prevention and lesion arrest in children younger than 6 years according to guidelines from the American Dental Association, American Academy of Paediatric Dentistry and US Preventive Services Task Force. ${ }^{2-4}$ In children younger than 6 years at high risk for dental caries, the recommendation is application
Strengths and limitations of this study

The study is based on in vitro and preclinical studies showing the study drug to treat infection with Streptococcus mutans, the primary pathogen in dental caries (tooth decay), to be safe.

- The primary components of the drug, povidone iodine and sodium fluoride were effective in reducing new dental caries when applied sequentially in two cohort studies.

- The study uses an active control but does not include a placebo because standard fluoride varnishes are considered standard of care.

- Because the study is in a single site, future multicentre studies will be required to confirm the hypothesised effect and allow for generalisation.

of NaF varnish every 3 months. ${ }^{2}$ Fluoride varnishes have been shown to be safe in young children. ${ }^{5}$

Topical fluoride alone, however, is not enough to thwart tooth decay in high-risk populations. Caries researchers for some time have suggested strategies that combine an antiseptic with topical fluoride to reduce or eliminate tooth surface colonisations by cariogenic bacteria in addition to topical fluoride which primarily remineralises enamel. ${ }^{6}$ Povidone iodine (PVP-I) is a widely used over-the-counter antiseptic and approved by the Food and Drug Administration (FDA) for intraoral and extraoral use. For the oral flora, iodine has preferential activities against streptococcal species, including Streptococcus mutans, pathogens implicated in the causation of dental caries. ${ }^{78}$ Moreover, iodine's effectiveness may last as long as 6 months. ${ }^{9}$

Studies have examined suppression of $S$. mutans by PVP-I in children with severe early childhood caries (S-ECC). In one study, the teeth of children 2-6 years of age with S-ECC were treated topically with PVP-I or saline following surgical dental treatment under general anaesthesia for S-ECC. ${ }^{10}$ Levels of $S$. 
mutans were significantly reduced up to 3 months in the PVP-I group $(\mathrm{p}<0.001)$. A second study demonstrated suppression of $S$. mutans up to 90 days in children with S-ECC who received a single application of PVP-I followed by a topical application of $1.23 \%$ acidulated phosphate fluoride foam and surgical elimination of caries lesions. ${ }^{11}$

A study of babies in Puerto Rico who were at high risk for S-ECC because they were all colonised by S. mutans and had decay-promoting feeding behaviours demonstrated that PVP-I applied bimonthly prevented the development of early tooth decay lesions in the maxillary primary incisors. ${ }^{12}$ In a randomised clinical trial, PVP-I applied bimonthly reduced recurrent dental decay assessed at 6 months following surgical treatment for S-ECC. ${ }^{13}$

Two prospective cohort studies tested the effect of sequentially applying PVP-I followed by $5 \% \mathrm{NaF}$ varnish. In these studies, children in one school setting received the combination treatment, and the outcome was compared with that of children in a similar school setting in the same community who received only application of the $5 \% \mathrm{NaF}$ varnish. There was no randomisation of children or schools. The first study assessed the effect of protecting erupting first permanent molars from developing dental caries in children $5-6$ years old ${ }^{14}$; the second studied the effect on the primary dentition of children 12-30 months. ${ }^{15}$ Both demonstrated that treatment with PVP-I reduced the incidence of new decay over $\mathrm{NaF}$ varnish alone $(\mathrm{p}<0.002$ and $\mathrm{p}<0.02$, respectively).

This phase II randomised controlled trial assesses the efficacy of a combination PVP-I and NaF dental varnish (test varnish) to determine if it is superior to a varnish containing only $\mathrm{NaF}$ (active control) in the prevention of new caries lesions.

\section{METHODS}

\section{Trial design}

This is a single-centre randomised, double-blind, active-controlled, parallel-group trial. The trial has been registered with ClinicalTrials.gov (NCT03082196) and is currently in the active phase. The protocol number is 2016-12-02.

\section{Research objectives and hypothesis}

The purpose of the study is to determine if the test varnish is superior to an active control varnish containing only $\mathrm{NaF}$ in the prevention of new caries lesions. Secondary objectives are to establish that the child has no more anxiety during the application of the test varnish than when the control varnish is applied and to document the safety of the varnish.

\section{Participant recruitment, enrolment and retention}

The study sites are early childhood education programmes at 10 schools in Pohnpei State, Federated States of Micronesia. Children attending these programs are the participants enrolled. The parent or legal guardian of the potential participant was approached at the school after drop off or prior to pick up by a study assistant who explained the study and obtained written informed consent. There were 299 consents. After the consent, a study assistant collected healthy history information, and the final decision whether to enrol the child was made by a study dentist after a review of the history. Seven children were excluded because they were too young. Enrolment began 22 March 2017 and was completed on 7 April 2017: 285 children were examined, and 284 aged 48-84 months old were randomised and received the first study treatment. The child who was examined and not randomised was determined not to be able to cooperate with the study treatment because of developmental delays.

The Micronesian islands are quite isolated, and children do not change schools or move from the islands readily. To maintain participation, we will regularly provide healthy snacks at the centres and hold end of year study parties for parents and children at the centres to reduce drop-outs. Families whose children completed the first treatment visit received a $\$ 35$ gift card to a local grocery store as an incentive. Families whose children complete a year in the study will receive another $\$ 35$ gift card; families whose children complete 2 years in the study will receive a third $\$ 35$ gift card. In addition, the children received a study backpack including a set of school supplies. To be more inclusive, all children in the programmes received a free study t-shirt, irrespective of whether they are enrolled in the study. The incentive levels were determined based on advice from the Department of Health.

The Western Institutional Review Board (designated IRB) and the Institutional Review Board of the College of Micronesia-FSM approved all study procedures. The FDA has issued IND 128835 covering this study. Protocol amendments are subject to approval of the IRB. Copies of the parent information sheet and consent forms are in the online supplementary appendix 1.

\section{Interventions}

The test varnish contains PVP-I and 5\% NaF (w/v). One and 2 years shelf life of the varnish has been established according to FDA Guidance for Industry Q1A(R2) Stability Testing of New Drug Substances and Products (Revision 2, November 2003). The active control varnish is the same NaF varnish without PVP-I. There is no difference in the test and control varnishes except for the PVP-I. Both varnishes were prepared by Cascade Custom Chemistry (Eugene, Oregon, USA). A maximum of $0.2 \mathrm{~mL}$ of varnish is applied topically to the teeth with a dental applicator. The varnish is applied every 3-4 months.

\section{Data collection}

Participants were examined clinically at enrolment. Follow-up examinations will be at 1 and 2 years.

\section{Outcome}

The primary outcome is the surface-level primary molar caries increment $\left(\mathrm{d}_{2-3} \mathrm{mfs} / \mathrm{DMFS}\right)$ at 2 years post baseline, but the incremental dental caries at 1 year will also be compared between the two interventions. The secondary 
outcome is the Facial Image Scale ${ }^{16}$ administered after the initial treatment and after the fifth treatment to gauge the child's anxiety in response to the treatment.

At baseline, two examiners were trained and calibrated according to National Institute of Dental and Craniofacial Research Early Childhood Caries Collaborative criteria, which are based on the WHO caries classification system. The children were visually examined using a mouth mirror and artificial light in a portable dental chair. No explorers were used. Examiners did not use dental radiographs. At follow-up, examiners will be recalibrated and will not have access to the results of the first examination to avoid detection bias. The data are collected on paper forms and transferred to secure computer files at the University of Washington. The methods for data entry, storage and handling are detailed in the manual of procedures.

\section{Sample size and power estimates}

Based on the caries levels reported by Tut and Milgrom ${ }^{14}$ and Chi $e t a l,{ }^{17}$ a conservative estimate for the coefficient of variation $(100 \times \mathrm{SD} /$ mean $)$ is $75 \%$. Using a two-sided 0.025 significance level to control the type I error for testing at 1 and 2 years post baseline, 120 participants per intervention are required to demonstrate a $30 \%$ or greater reduction in caries in the test group as compared with the active control varnish group with $90 \%$ power. ${ }^{18} 19$ A $30 \%$ reduction by the sequential topical treatment of PVP-I liquid followed by $\mathrm{NaF}$ varnish versus $\mathrm{NaF}$ varnish alone was reported by Milgrom $e t a l^{15}$ for any new caries lesions (\%), and a greater reduction would be expected in the amount of new caries lesions in a high-risk caries population. To accommodate for attrition (up to 15\%), the target enrolment was to be at least 280 participants, although attrition is expected to be very low in this study population.

\section{Randomisation and blinding}

Children were randomly assigned in 1:1 ratio to the test or control varnish. The randomisation was stratified on school and used randomly permuted blocks of size 2 and 4 selected with $3 / 4$ and $1 / 4$ probability to maintain balanced assignment within each school and concealment of the varnish assignment. The study biostatistician created the randomisation lists for each school using the 'sample' function of the R statistical software (V.3.3.0; The R Foundation for Statistical Computing, 2016). For the first varnish application, lists were prepared for A and $\mathrm{B}$ assignments separately for each school, and these lists were used when the varnish is applied. Small groups of children from the lists were called from their classroom for varnish application. The drug containers were marked clearly, and in each case, a second staff member verified that the participant received the correct varnish. The same procedure will be followed for the additional treatments.

All study personnel except for designated personnel in the data centre are blinded to the participant treatment assignment. In order to maintain blinding, the caries scoring examinations preceded the application of test varnishes at baseline. The staff members applying these varnishes do not perform the caries scoring examinations in order to eliminate examiner bias and ensure blinding to treatment assignment.

\section{Planned analysis}

\section{Analysis of primary outcome}

The primary analysis will be conducted in accordance with the intention-to-treat principle, and multiple imputation procedures will be used to account for missing caries information. ${ }^{20}$ For the primary outcome, incremental dental caries based on $\mathrm{d}_{2-3} \mathrm{mfs} / \mathrm{DMFS}$, the unit of analysis will be the child, and the mean dental caries increment will be compared between the two interventions separately at 1 and 2 years post baseline using a two-sample t-test assuming unequal variances. This approach assumes the distribution of dental caries increment is approximately symmetrical, which is often true in high-risk caries populations. In the case of non-symmetry, log-linear regression using robust SEs, which can accommodate count outcomes with skewed distribution, will be used to compare the caries rates between the two interventions. ${ }^{20}$ Covariate-adjusted linear (or log-linear) regression models using robust SEs will be used to compare dental caries increment, adjusting for baseline dental caries, stratification variables (education centre and school), gender and age of the participant. ${ }^{21}$ Analyses will be performed using R V.3.3.0 (The R Foundation for Statistical Computing, 2016) and SAS V.9.4 (SAS Institute).

\section{Analysis of secondary outcome}

The secondary outcome will be the child response on the Facial Image Scale (1-5) to the initial varnish application at baseline and at the fifth varnish application at the end of year 1 . Average score will be compared between the two interventions separately at baseline and year 1 using a two-sample t-test assuming unequal variances. Additional comparisons will be performed that adjust for child age, child gender and school using analysis of covariance.

\section{Harms}

There are 24-hour safety checks on the health of all participants and an onsite medical monitor. Severe adverse events that might result in a child being withdrawn or the study modified or discontinued are reported immediately to the IRB and to the FDA, as required under US law. The medical monitor has the ability to unblind the treatment assignment for a particular participant in the event of a severe adverse event. The sponsor has obtained clinical trial liability insurance that covers any medical costs for a participant in the event of an adverse event related to the study drug.

\section{Auditing}

The sponsor has appointed an independent regulatory monitor who is registered with US FDA. The monitor 
made the site initiation visit on 28-29 March 2017 and will audit the onsite records each year and at study close.

\section{Dissemination}

Results of the trial will be posted on ClinicalTrials.gov, reported at the Annual Meeting of the International Association for Dental Research and published in a scholarly journal.

\section{Discussion}

Dental caries, a bacterial disease most strongly associated with the mutans group of streptococci (here, S. mutans), is exacerbated by a high-carbohydrate diet. It results in dissolution of mineralised tooth structure by acids produced by dental plaque bacteria. ${ }^{22}{ }^{23}$ Untreated caries lesions can result in pain, regional and systemic infections; impaired oral function; and other personal and population problems. The US Surgeon General's Report noted, 'dental caries is the single most common chronic childhood disease'. ${ }^{24}$

While critical in the prevention of dental caries for decades, topical fluoride by itself is not enough to prevent tooth decay in high-risk populations. ${ }^{625}$ The proposed work is innovative because the test varnish takes advantage of possible biological synergies of an antimicrobial, PVP-I, and a tooth surface remineralising agent, NaF, in a single non-invasive procedure that depends on minimal expenditure. If efficacious, it could change the standard of care for caries prevention and arrest.

Contributors PM, LM and JMT conceived of the study and wrote the original protocol. OT, MR and MG developed methods for recruitment and procedures and contributed to the revision of the protocol. All of the authors are part of the clinical trial implementation team. All of the others contributed to the final editing of the manuscript and agreed to its contents.

Funding This trial is funded by Advantage Silver Dental Arrest, 442 SW Umatilla, Suite 200, Redmond, OR 97756, USA. Planning funds were provided by a grant from the National Institute of Oral and Craniofacial Research, NIH (grant \# 2R44 DE022986-02). PM is a director of Advantage Silver Dental Arrest. OT and JT are consultants to Advantage. Dr Tut is the principal investigator of the trial. The other authors declare no conflict of interest.

Competing interests PM is a director of Advantage Silver Dental Arrest, LLC. OT and JT are consultants to Advantage. Dr. Tut is the principal investigator of the trial. The other authors declare no conflict of interest.

Patient consent Obtained.

Ethics approval Western Institutional Review Board.

Provenance and peer review Not commissioned; peer reviewed for ethical and funding approval prior to submission.

Data sharing statement The final dataset, including all forms, protocol and manual of procedures, will be maintained at the University of Washington and the offices of the sponsor. A copy will be filed with the US Food and Drug Administration. After filing, a public dataset will be available from the sponsor.

Open Access This is an Open Access article distributed in accordance with the Creative Commons Attribution Non Commercial (CC BY-NC 4.0) license, which permits others to distribute, remix, adapt, build upon this work non-commercially, and license their derivative works on different terms, provided the original work is properly cited and the use is non-commercial. See: http://creativecommons.org/ licenses/by-nc/4.0/

(c) Article author(s) (or their employer(s) unless otherwise stated in the text of the article) 2017. All rights reserved. No commercial use is permitted unless otherwise expressly granted.

\section{REFERENCES}

1. Gao SS, Zhang S, Mei ML, et al. Caries remineralisation and arresting effect in children by professionally applied fluoride treatment-a systematic review. BMC Oral Health 2016;16:12.

2. Weyant RJ, Tracy SL, Anselmo TT, et al. Topical fluoride for caries prevention: executive summary of the updated clinical recommendations and supporting systematic review. J Am Dent Assoc 2013;144:1279-91.

3. American Academy of Pediatric Dentistry. Guideline on periodicity of examination, preventive dental services, anticipatory guidance/ counseling, and oral treatment for infants, children, and adolescents. Pediatr Dent 2013:35:E148-56.

4. Moyer VA. US Preventive Services Task Force. Prevention of dental caries in children from birth through age 5 years: US Preventive Services Task Force recommendation statement. Pediatrics 2014;133:1102-11.

5. Milgrom P, Taves DM, Kim AS, et al. Pharmacokinetics of fluoride in toddlers after application of $5 \%$ sodium fluoride dental varnish. Pediatrics 2014;134:e870-e874.

6. Milgrom P, Zero DT, Tanzer JM. An examination of the advances in science and technology of prevention of tooth decay in young children since the Surgeon General's Report on Oral Health. Acad Pediatr 2009;9:404-9.

7. Tam A, Shemesh M, Wormser U, et al. Effect of different iodine formulations on the expression and activity of Streptococcus mutans glucosyltransferase and fructosyltransferase in biofilm and planktonic environments. J Antimicrob Chemother 2006;57:865-71.

8. Furiga A, Dols-Lafargue M, Heyraud A, et al. Effect of antiplaque compounds and mouthrinses on the activity of glucosyltransferases from Streptococcus sobrinus and insoluble glucan production. Oral Microbiol Immunol 2008;23:391-400.

9. Caufield PW, Gibbons RJ. Suppression of Streptococcus mutans in the mouths of humans by a dental prophylaxis and topically-applied iodine. J Dent Res 1979;58:1317-26.

10. Zhan L, Featherstone JD, Gansky SA, et al. Antibacterial treatment needed for severe early childhood caries. J Public Health Dent 2006;66:174-9.

11. Berkowitz RJ, Koo H, McDermott MP, et al. Adjunctive chemotherapeutic suppression of mutans streptococci in the setting of severe early childhood caries: an exploratory study. J Public Health Dent 2009;69:163-7.

12. Lopez L, Berkowitz R, Spiekerman C, et al. Topical antimicrobial therapy in the prevention of early childhood caries: a follow-up report. Pediatr Dent 2002;24:204-6.

13. Amin MS, Harrison RL, Benton TS, et al. Effect of povidone-iodine on Streptococcus mutans in children with extensive dental caries. Pediatr Dent 2004;26:5-10.

14. Tut OK, Milgrom PM. Topical iodine and fluoride varnish combined is more effective than fluoride varnish alone for protecting erupting first permanent molars: a retrospective cohort study. J Public Health Dent 2010;70:249-52.

15. Milgrom PM, Tut OK, Mancl LA. Topical iodine and fluoride varnish effectiveness in the primary dentition: a quasi-experimental study. $J$ Dent Child 2011;78:143-7.

16. Buchanan $\mathrm{H}$, Niven N. Validation of a Facial image Scale to assess child dental anxiety. Int J Paediatr Dent 2002;12:47-52.

17. Chi DL, Tut O, Milgrom P. Cluster-randomized xylitol toothpaste trial for early childhood caries prevention. J Dent Child 2014;81:27-32.

18. Van Belle G, Martin DC. Sample size as a function of coefficient of variation and ratio of means. Am Stat 1993;47:165-7.

19 . R package version 1.1-3. 2015. https://CRAN.R-project.org/ package=pwr (Accessed on December 1, 2016).

20. Schafer JL. Analysis of Incomplete Multivariate Data. New York, NY. USA: Chapman \& Hall, 1997.

21. Hardin JW, Hilbe JM. Chapman and Hall/CRC. Generalized Estimating Equations. New York, 2003.

22. Loesche WJ. Role of Streptococcus mutans in human dental decay. Microbiol Rev 1986;50:353-80.

23. Tanzer JM, Livingston J, Thompson AM. The microbiology of primary dental caries in humans. J Dent Educ 2001;65:1028-37.

24. U.S. Department of Health and Human Services (USDHHS). Oral Health in America: A Report of theSurgeon General. Rockville, MD: HHS, National Institutes of Health, National Institute of Dental and Craniofacial Research, 2000.

25. Weinstein P, Spiekerman C, Milgrom P. Randomized equivalence trial of intensive and semiannual applications of fluoride varnish in the primary dentition. Caries Res 2009;43:484-90. 